\title{
Increased 3-hydroxypropionic acid production from glycerol, by modification of central metabolism in Escherichia coli
}

\author{
Kento Tokuyama', Satoshi Ohno ${ }^{1}$, Katsunori Yoshikawa', Takashi Hirasawa ${ }^{1,2}$, Shotaro Tanaka',
} Chikara Furusawa ${ }^{1,3}$ and Hiroshi Shimizu ${ }^{1 *}$

\begin{abstract}
Background: 3-hydroxypropionic acid (3HP) is an important chemical precursor for the production of bioplastics. Microbial production of $3 \mathrm{HP}$ from glycerol has previously been developed through the optimization of culture conditions and the 3HP biosynthesis pathway. In this study, a novel strategy for improving 3HP production in Escherichia coli was investigated by the modification of central metabolism based on a genome-scale metabolic model and experimental validation.

Results: Metabolic simulation identified the double knockout of tpiA and zwf as a candidate for improving 3HP production. A 3HP-producing strain was constructed by the expression of glycerol dehydratase and aldehyde dehydrogenase. The double knockout of tpiA and zwf increased the percentage carbon-molar yield (C-mol\%) of $3 \mathrm{HP}$ on consumed glycerol 4.4-fold (20.1 $\pm 9.2 \mathrm{C}$-mol\%), compared to the parental strain. Increased extracellular methylglyoxal concentrations in the $\triangle t p i A \Delta z w f$ strain indicated that glycerol catabolism was occurring through the methylglyoxal pathway, which converts dihydroxyacetone phosphate to pyruvate, as predicted by the metabolic model. Since the $\triangle t p i A \Delta z w f$ strain produced abundant 1,3-propanediol as a major byproduct (37.7 $\pm 13.2 \mathrm{C}$-mol\%), yqhD, which encodes an enzyme involved in the production of 1,3-propanediol, was disrupted in the $\Delta t p i A \Delta z w f$ strain. The $3 \mathrm{HP}$ yield of the $\triangle$ tpiA $\triangle z w f \Delta y q h D$ strain (33.9 $1.2 \mathrm{C}$-mol\%) was increased 1.7-fold further compared to the $\triangle t p i A \Delta z w f$ strain and by 7.4 -fold compared to the parental strain.
\end{abstract}

Conclusion: This study successfully increased 3HP production by 7.4-fold in the $\triangle \operatorname{tpiA} \triangle z w f \Delta y q h D E$. coli strain by the modification of the central metabolism, based on metabolic simulation and experimental validation of engineered strains.

Keywords: 3-hydroxypropionic acid, Escherichia coli, Glycerol, Genome-scale metabolic model, Central metabolism

\section{Background}

3-hydroxypropionic acid (3HP) has recently attracted attention due to its availability as a precursor of valuable chemicals such as acrylic acid, $\beta$-propiolactone, and malonic acid $[1,2]$. Its polymerized form, poly $(3 \mathrm{HP})$, is a promising alternative to petrochemical-derived plastic $[3,4]$. Because of this superior industrial availability, 3HP was designated as one of the top value-added chemicals produced by biomass, by the U.S. Department of Energy

\footnotetext{
* Correspondence: shimizu@ist.osaka-u.ac.jp

'Department of Bioinformatic Engineering, Graduate School of Information Science and Technology, Osaka University, 1-5 Yamadaoka, Suita, Osaka 565-0871, Japan

Full list of author information is available at the end of the article
}

[5,6]. In the commercial bioproduction process, the substrate has a significant impact on production cost. Glycerol is a potential substrate for bioproduction considering that the recent expansion of biodiesel production has caused a surplus of glycerol as its byproduct, and a subsequent decrease in the price of glycerol [7-9].

The microbial production of 3HP from glycerol has been developed using a natural 3HP producer, Klebsiella pneumoniae [10-13]. Expression of the heterologous glycerol dehydratase and aldehyde dehydrogenase enabled $3 \mathrm{HP}$ to be produced in the non-natural 3HP producers Pseudomonas denitrificans [14,15] and Escherichia coli 
[16-19]. To date, various studies have reported increased 3HP production [10-19]. For example, Rathnasingh et al. optimized the expression level of each enzyme in this pathway in E. coli [17]. Ashok et al. deleted dhaT and $y q h D$ to reduce the production of byproducts in $K$. pneumoniae [12]. Kim et al. modified glycerol metabolism in 3HP-producing E. coli and developed fed-batch cultivation with simultaneous feeding of glycerol and glucose [19]. As described above, most previous studies focused on the optimization of metabolic reactions from glycerol to $3 \mathrm{HP}$ and culture conditions. Considering the whole metabolic network, modification of other pathways, as well as the biosynthetic pathway of the target product, is also a key strategy for increasing the metabolic flux, leading to enhanced target production. For example, target production can be enhanced by improving the redox balance and reducing byproduct formation via gene knockout and overexpression [12,20].

Recently, in silico metabolic simulation has been developed to consider whole metabolic networks. A genome-scale metabolic model, which includes most of the metabolic reactions of the cell [21-23], can estimate the flux distribution of the whole metabolic network using flux balance analysis (FBA) [24,25] by assuming the steady states of metabolic reactions and maximizing objective functions such as cell growth [26,27]. This method can be used to simulate the effects of gene modifications on target production and identify candidate genes for metabolic engineering [28-30]. Successful improvements in target production have been reported in many such simulations [31-33].

The introduction of heterologous genes and gene manipulations does not always produce the expected growth behavior and target production in engineered strains. Experimental validation of engineered strains provides useful information about the actual metabolic state of a cell such as bottleneck reactions, growth inhibition factors, and redox imbalance [34-36]. A strain engineered on the basis of metabolic simulation should be evaluated experimentally to develop the next strategy for improving target production.

The current study aimed to enhance $3 \mathrm{HP}$ production from glycerol in E. coli by focusing on the whole metabolic network. Metabolic pathway modification was designed by integrating in silico gene-knockout simulation with the experimental validation of engineered strains. The genome-scale metabolic model of $E$. coli was used to design a metabolic network for increased 3HP production. Metabolic simulation identified two candidate genes to be deleted, tpiA and $z w f$, which are involved in central metabolism. Based on the simulation, knockout strains were constructed and $3 \mathrm{HP}$ production was successfully increased, as predicted. In addition, a gene, $y q h D$, related to the biosynthesis pathway of a major byproduct, 1,3-propanediol (1,3-PDO), was deleted to further increase $3 \mathrm{HP}$ production.

\section{Results and discussion}

\section{Construction of a 3HP-producing strain in E. coli}

The 3HP biosynthetic pathway from glycerol consists of two reactions: the dehydration of glycerol to 3hydroxypropionaldehyde (3HPA), catalyzed by glycerol dehydratase, and the oxidation of 3HPA to 3HP, catalyzed by aldehyde dehydrogenase [16]. Since E. coli does not possess the $3 \mathrm{HP}$ biosynthetic pathway, the $3 \mathrm{HP}$-producing strain (TK52) was constructed by the overexpression of $d h a B$ and $g d r A B$, which encode for glycerol dehydratase and glycerol dehydratase reactivase (from $K$. pneumoniae), respectively, and ald $H$, which encodes for aldehyde dehydrogenase (from E. coli), as described in a previous study [17]. TK52 was cultivated in M9 medium in a Sakaguchi flask and 3HP was produced at $4.6 \pm 0.8 \%$ carbon-molar yield of 3HP on consumed glycerol (C-mol\%) (Figure 1A-B and Table 1). Acetate was produced as a major byproduct $(9.9 \pm 1.8 \mathrm{C}$-mol\%) and small yields of 1,3-PDO (0.8 $\pm 0.4 \mathrm{C}$-mol\%) and succinate $(0.9 \pm 0.1$ $\mathrm{C}$-mol\%) were also produced. Ethanol and formate were not detected in this strain and other strains that were constructed. Although E. coli does not possess 1,3-PDO biosynthetic pathways, the introduction of $d h a B$ for $3 \mathrm{HP}$ production enabled production of 1,3-PDO as follows: glycerol dehydratase converts glycerol to 3HPA, and an endogenous alcohol dehydrogenase (encoded for by $y q h D$ ) further converts 3HPA to 1,3-PDO [13,32].

\section{Gene knockout simulation for 3HP production}

Metabolic simulation was carried out to improve 3HP production by considering the whole metabolic network. A genome-scale metabolic model of E. coli, iAF1260, which includes 2,077 metabolic and transport reactions and 1,038 unique metabolites [21], was employed with FBA $[24,25]$ to simulate $3 \mathrm{HP}$ production in E. coli. Since the $3 \mathrm{HP}$ biosynthesis pathway does not exist in $E$. coli, seven reactions involved in the $3 \mathrm{HP}$ biosynthetic pathway were added to the iAF1260 model (Table 2), which was subsequently referred to as the iAF1260-3HP model. Using the iAF1260-3HP model, multiple gene knockout simulations were performed to identify candidate genes that could enhance $3 \mathrm{HP}$ production when deleted. For simulation parameters, glycerol was used as the sole carbon source, and its uptake rate (GUR) was set to $15 \mathrm{mmol} /(\mathrm{g}$ dry cell $(\mathrm{DC}) \bullet \mathrm{h})$. The oxygen uptake rate (OUR) was set to $10 \mathrm{mmol} /(\mathrm{g} \mathrm{DC} \bullet \mathrm{h})$, which corresponds to a microaerobic condition.

In single-gene knockout simulations, solutions in which the metabolic flux of $3 \mathrm{HP}$ production was higher than zero were not obtained. Double-gene knockout simulations identified some candidate genes that when 

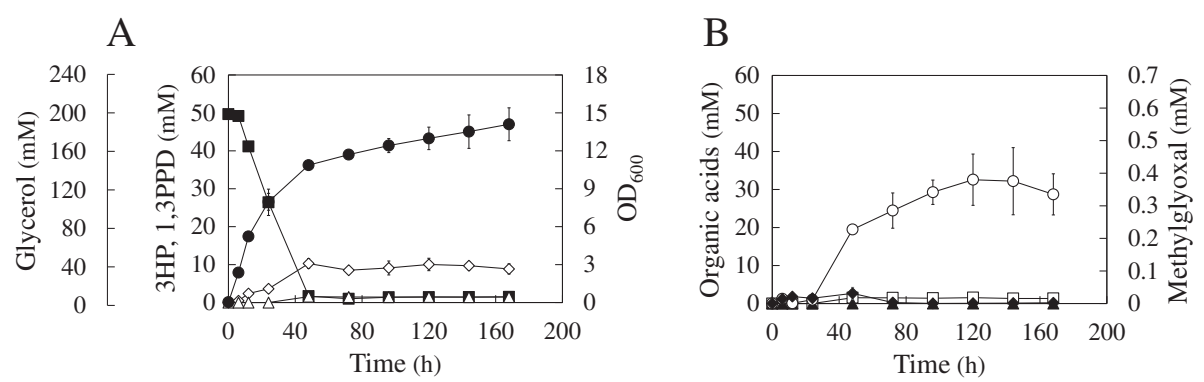

C

$\mathrm{D}$
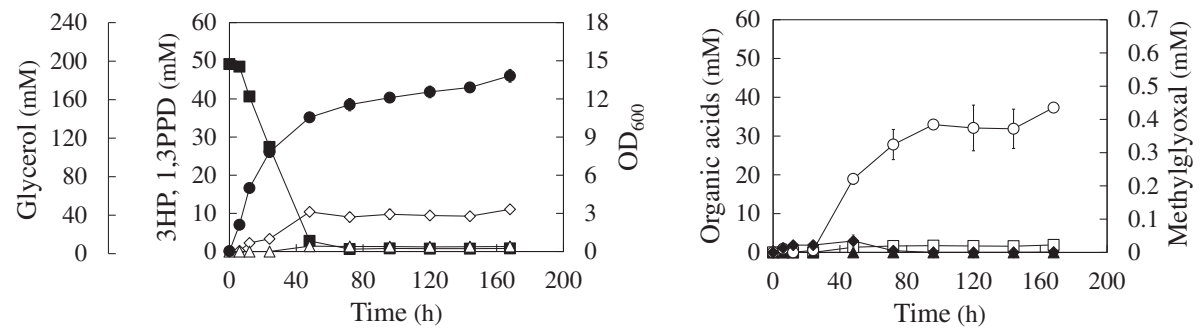

$\mathrm{E}$

\section{F}
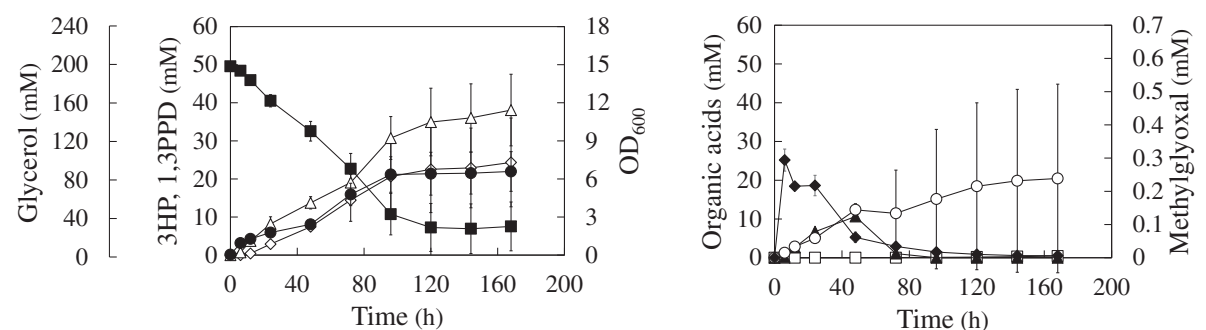

G

$\mathrm{H}$
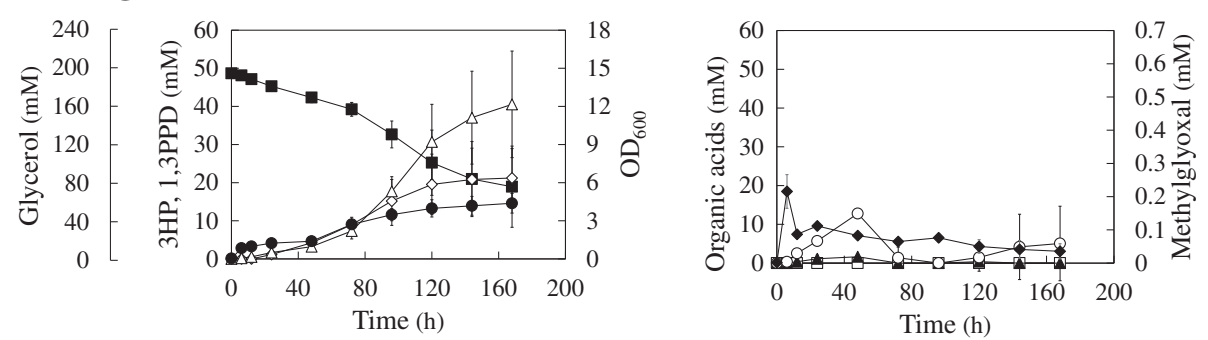

$\mathrm{J}$
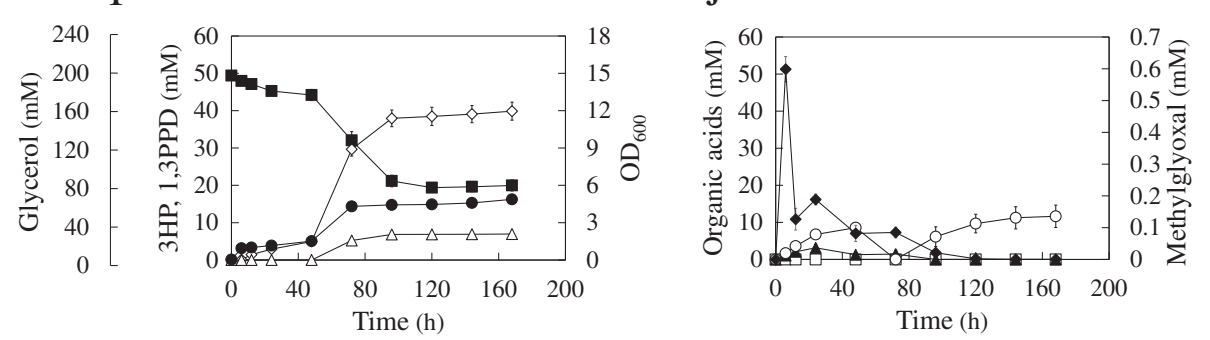

Figure 1 Culture results of the 3HP-producing strains. The culture results of the strains TK52 (A, B), TK52Z (C, D), TK52t (E, F), TK52tz (G, H), and TK52tzy $(\mathbf{I}, \mathbf{J})$ are shown. Open diamond, 3HP; closed square, glycerol; open triangle, 1,3-PDO; closed circle, OD 600 ; open circle, acetate; open square, succinate; closed triangle, lactate; closed diamond, methylglyoxal. Error bars represent standard deviation of triplicate experiments in TK52 and TK52Z strains, and of nine replicate experiments in other strains. Some of the error bars are smaller than the symbols. 
Table 1 Summary of the experimental results

\begin{tabular}{|c|c|c|c|c|c|c|c|}
\hline & TK52 & TK52z & TK52t & TK52tz & TK52tzy & $\begin{array}{l}\text { Simulation }^{* 4} \\
\text { iAF1260-3HP }\end{array}$ & $\begin{array}{c}\text { Simulation }^{* 4} \\
\Delta \text { tpiA } \Delta z w f\end{array}$ \\
\hline \multirow[t]{2}{*}{ Specific growth rate $(1 / h)^{* 1}$} & $0.73 \pm 0.00$ & $0.71 \pm 0.00$ & 1st: $0.55 \pm 0.01$ & 1st: $0.54 \pm 0.01$ & 1st: $0.56 \pm 0.01$ & & \\
\hline & & & 2nd: $0.03 \pm 0.01$ & 2nd: $0.03 \pm 0.00$ & 2nd: $0.04 \pm 0.01$ & & \\
\hline Maximum $3 \mathrm{HP}$ production rate $(\mathrm{mmol} /(\mathrm{g} \mathrm{DC} \bullet \mathrm{h}))^{* 2}$ & $0.08 \pm 0.02$ & $0.09 \pm 0.01$ & $0.22 \pm 0.14$ & $0.27 \pm 0.11$ & $0.94 \pm 0.05$ & & \\
\hline Consumed glycerol $(\mathrm{mM})^{* 3}$ & $192.7 \pm 5.0$ & $193.2 \pm 2.4$ & $168.2 \pm 24.4$ & $119.0 \pm 44.6$ & $117.6 \pm 3.9$ & & \\
\hline \multirow[t]{2}{*}{ Biomass $(\mathrm{C}-\mathrm{mol} \%)^{* 3}$} & $37.0 \pm 3.5$ & $36.1 \pm 1.4$ & $19.6 \pm 3.2$ & $21.7 \pm 11.0$ & $20.8 \pm 1.2$ & 47.7 & 22.8 \\
\hline & $(5.2 \pm 0.5)$ & $(5.1 \pm 0.2)$ & $(2.4 \pm 0.6)$ & $(1.6 \pm 0.3)$ & $(1.8 \pm 0.1)$ & & \\
\hline \multirow[t]{2}{*}{$3 \mathrm{HP}(\mathrm{C}-\mathrm{mol} \%)^{* 3}$} & $4.6 \pm 0.8$ & $5.7 \pm 0.6$ & $14.7 \pm 7.0$ & $20.1 \pm 9.2$ & $33.9 \pm 1.2$ & 0 & 70.5 \\
\hline & $(8.9 \pm 1.3)$ & $(11.1 \pm 1.0)$ & $(24.3 \pm 11.7)$ & $(21.2 \pm 7.7)$ & $(39.9 \pm 2.4)$ & & \\
\hline \multirow[t]{2}{*}{ 1,3-PDO $(\mathrm{C}-\mathrm{mol} \%)^{* 3}$} & $0.8 \pm 0.4$ & $0.7 \pm 0.2$ & $22.9 \pm 5.2$ & $37.7 \pm 13.2$ & $5.9 \pm 0.5$ & 0 & 0 \\
\hline & $(1.5 \pm 0.8)$ & $(1.3 \pm 0.3)$ & $(38.1 \pm 9.4)$ & $(40.5 \pm 14.0)$ & $(7.0 \pm 0.7)$ & & \\
\hline \multirow[t]{2}{*}{ Succinate $(\mathrm{C}-\mathrm{mol} \%)^{* 3}$} & $0.9 \pm 0.1$ & $1.3 \pm 0.2$ & $0.3 \pm 0.3$ & 0 & 0 & 0 & 0 \\
\hline & $(1.3 \pm 0.2)$ & $(1.9 \pm 0.2)$ & $(0.4 \pm 0.3)$ & (0) & (0) & & \\
\hline \multirow[t]{2}{*}{ Acetate $(\mathrm{C}-\mathrm{mol} \%)^{* 3}$} & $9.9 \pm 1.8$ & $12.9 \pm 0.2$ & $8.2 \pm 9.9$ & $2.2 \pm 4.1$ & $6.6 \pm 1.9$ & 27.4 & 0 \\
\hline & $(28.7 \pm 5.4)$ & $(37.3 \pm 1.1)$ & $(20.5 \pm 24.3)$ & $(5.1 \pm 9.6)$ & $(11.6 \pm 3.0)$ & & \\
\hline Maximum methylglyoxal concentration (mM) & $0.03 \pm 0.02$ & $0.03 \pm 0.02$ & $0.29 \pm 0.02$ & $0.22 \pm 0.02$ & $0.60 \pm 0.02$ & & \\
\hline
\end{tabular}

${ }^{{ }^{1}}$ Specific growth rates were calculated using $\mathrm{OD}_{600}$ at 0-6 $\mathrm{h}$ for the TK52 and TK52z strains. For the TK52t, TK52tz and TK52tzy strains, specific growth rates during the 1st and 2nd growth phases were calculated using the $\mathrm{OD}_{600}$ at $0-6 \mathrm{~h}$ and $48-72 \mathrm{~h}$, respectively.

${ }^{*}$ Maximum $3 \mathrm{HP}$ production rates wer calculated from the data at $24-48 \mathrm{~h}$ for the TK52 and TK52z strains, and at 48-72 $\mathrm{h}$ for other strains.

${ }^{*} \mathrm{C}$-mol\% was calculated from the carbon-mol of the product per carbon-mol of the consumed glycerol. The values in the parentheses indicate the final concentrations of biomass ( $\mathrm{g} / \mathrm{L}$ ) and products ( $\mathrm{mM}$ ). Standard deviations were obtained from triplicate experiments in the TK52 and TK52z strains, and from 9 replicate experiments in other strains. For calculation of biomass yield, OD 600 was converted into dry cell weight using the conversion factor 0.37 $\mathrm{g} \mathrm{DC/L}$, and carbon-mol in the biomass was calculated based on the biomass composition described in the iAF1260 model [21].

${ }^{* 4}$ Metabolic simulation was performed with following parameters: GUR was $15 \mathrm{mmol} /(\mathrm{g} D C \bullet \mathrm{h})$ and OUR was $10 \mathrm{mmol} /(\mathrm{g} \mathrm{DC} \bullet \mathrm{h})$. 
Table 2 The metabolic reactions added to the iAF1260 model

\begin{tabular}{lll}
\hline Reaction name & Metabolic reaction & EC number \\
\hline Glycerol dehydratase & $\mathrm{Glycerol} \rightarrow 3 \mathrm{HPA}+\mathrm{H}_{2} \mathrm{O}$ & 4.2 .1 .30 \\
3HPA dehydrogenase & $3 \mathrm{HPA}+\mathrm{NAD}^{+}+\mathrm{H}_{2} \mathrm{O} \rightarrow 3 \mathrm{HP}+\mathrm{NADH}+2 \mathrm{H}^{+}$ & 1.2 .1 .3 \\
3HP transporter & $3 \mathrm{HP}+\mathrm{H}^{+}+3 \mathrm{HP}[\mathrm{e}]+\mathrm{H}^{+}[\mathrm{e}]$ & - \\
3HP exchange & $3 \mathrm{HP}[\mathrm{e}] \rightarrow$ & - \\
1,3-PDO oxidoreductase & $3 \mathrm{HPA}+\mathrm{NADPH}+\mathrm{H}^{+} \rightarrow 1,3 \mathrm{PDO}+\mathrm{NADP}^{+}$ & 1.1 .1 .202 \\
1,3-PDO transporter & $1,3-\mathrm{PDO} \rightarrow 1,3-\mathrm{PDO}[\mathrm{e}]$ & - \\
1,3-PDO exchange & $1,3-\mathrm{PDO}[\mathrm{e}] \rightarrow$ & - \\
\hline
\end{tabular}

Metabolites with "[e]" indicate extracellular metabolites.

deleted together could enhance $3 \mathrm{HP}$ production (Table 3 ). Among these, $\Delta t p i A \Delta p g i, \Delta t p i A \Delta z w f$, and $\Delta t p i A \Delta e d d$ models displayed the highest carbon-mol yield of $3 \mathrm{HP}$ on glycerol (70.5 C-mol\%). In this study, we focused on the double knockout of tpiA and $z w f$ for further analysis. tpiA encodes for triosephosphate isomerase, which converts dihydroxyacetone phosphate (DHAP) to glyceraldehyde3-phosphate (GAP). zwf codes for glucose-6-phosphate-1dehydrogenase, which converts glucose-6-phosphate to 6-phospho-glucono-1,5-lactone.

The simulated flux distributions of the iAF1260-3HP, $\Delta t p i A, \Delta z w f$, and $\Delta t p i A \Delta z w f$ models are shown in Figure 2. In the iAF1260-3HP model, a high flux of glycolysis was predicted and ATP required for cell growth was mainly produced by glycolysis and the respiratory chain (Figure 2A). The $\Delta z w f$ model exhibited the same flux distribution as the iAF1260-3HP model (Figure 2B) because the flux of the glucose-6-phosphate1-dehydrogenase reaction was zero in the iAF1260-3HP model (Figure 2A). In the $\Delta t p i A$ model, glycerol was mainly catabolized through the Entner-Doudoroff pathway via dihydroxyacetone (DHA) and entered into gluconeogenesis and the TCA cycle. The flux into glycolysis was blocked by the inability to generate GAP from DHAP because the amount of GAP consumed by the fructose 6-phosphate aldolase reaction, which converts DHA and GAP to fructose 6-phosphate, was equal to the amount of GAP produced by the Entner-Doudoroff pathway (Figure 2C).

In the $\Delta t p i A \Delta z w f$ model, the $z w f$ knockout blocked flux into the Entner-Doudoroff pathway, which was

Table 3 Knockout candidate genes for enhancing 3HP production obtained by gene knockout simulation

\begin{tabular}{|c|c|c|}
\hline Knockout genes & 3HP yield (C-mol\%) & $\begin{array}{l}\text { Specific growth rate } \\
\text { relative to wild-type }\end{array}$ \\
\hline iAF1260-3HP & 0 & $100 \%$ \\
\hline$\triangle t p i A \Delta z w f$ & 70.5 & $48 \%$ \\
\hline$\Delta t p i A \Delta p g i$ & 70.5 & $48 \%$ \\
\hline$\Delta t p i A \Delta e d d$ & 70.5 & $48 \%$ \\
\hline$\triangle t p i A \Delta g l d A$ & 69.0 & $47 \%$ \\
\hline$\triangle t p i A \Delta f s a B$ & 69.0 & $47 \%$ \\
\hline
\end{tabular}

active in the $\triangle t p i A$ model. This resulted in glycerol catabolism through the glycerol kinase reaction, which converts glycerol to glycerol-3-phosphate, and the methylglyoxal pathway, in which DHAP is converted to pyruvate via methylglyoxal. $3 \mathrm{HP}$ was produced instead of acetate in the $\Delta t p i A \Delta z w f$ model. In the $\Delta t p i A$ model, acetate production was preferred since ATP was also generated. However, in the $\Delta t p i A \Delta z w f$ model, ATP was consumed by the glycerol kinase reaction, which is why total ATP was not generated by acetate production from glycerol. When the OUR was limited, 3HP production was increased, instead of acetate production, to balance the reduced capacity of the respiratory chain due to the reduction in $\mathrm{NADH}$ generation by $3 \mathrm{HP}$ production from glycerol (Figure 2D).

\section{HP production based on the metabolic simulation}

Based on the results from the metabolic simulation, both tpiA and $z w f$ were disrupted in the TK52 strain, generating strain TK52tz. The 3HP yield of TK52tz was successfully increased 4.4-fold relative to TK52 (20.1 \pm 9.2 C-mol\%) (Figure 1G-H and Table 1). TK52tz exhibited a two-step growth phase that was not observed in TK52, with specific growth rates of $0.541 / \mathrm{h}$ in the first growth phase $(0-6 \mathrm{~h})$ and $0.03 \mathrm{l} / \mathrm{h}$ in the second growth phase (48-72 h). $3 \mathrm{HP}$ was mainly produced in the second growth phase with the consumption of acetate and lactate that was produced prior to the second growth phase. The growth rate of TK52tz was decreased compared to TK52 (0.73 1/h), and glycerol was not completely consumed in TK52tz. Acetate and 1,3-PDO were produced as byproducts. Although 1,3-PDO production was not predicted by metabolic simulation, the experimental results of TK52tz were consistent with the simulation results, including the improvement in 3HP yield, the decrease in growth rate, and the lack of succinate production. This indicated that metabolic modeling is an effective strategy for the improvement of target production.

Based on the metabolic simulation, glycerol was predicted to be catabolized in TK52tz through the methylglyoxal pathway, which converts DHAP to pyruvate. This pathway is not usually active in $E$. coli due to allosteric inhibition by 


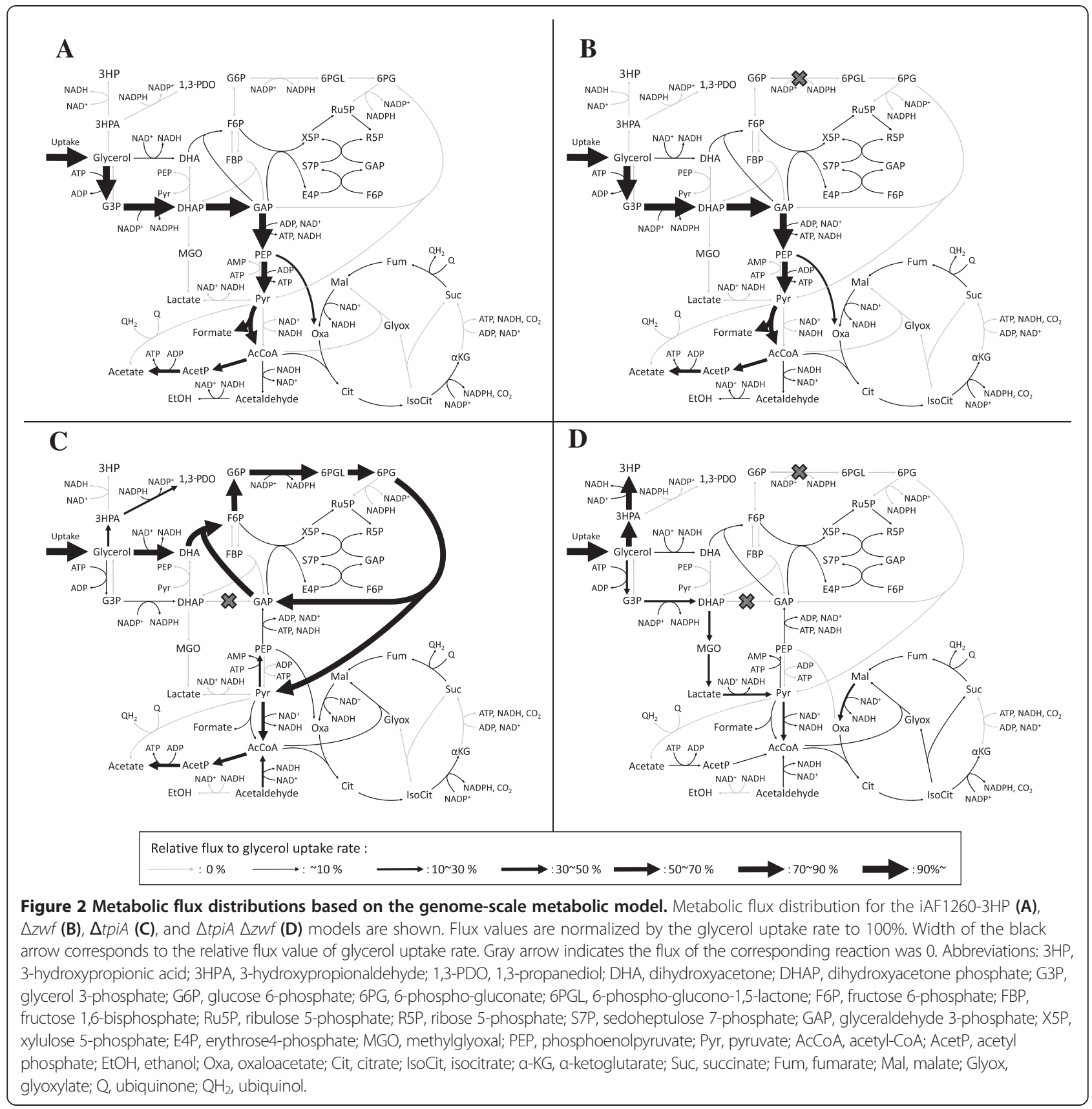

inorganic pyrophosphate and the low activity of enzymes involved in this pathway $[37,38]$. The extracellular concentration of methylglyoxal, an intermediate of the pathway, was increased significantly in TK52tz $(0.22 \mathrm{mM}$ at maximum) compared with TK52 (0.03 $\mathrm{mM}$ at maximum). This suggested that flux into the methylglyoxal pathway was increased as predicted, and this pathway might be a bottleneck for glycerol catabolism. This could result in decreased growth and incomplete glycerol consumption because methylglyoxal is a toxic cellular electrophile that reacts with the nucleophilic centers of macromolecules such as DNA, RNA, and protein [39].
Abundant production of 1,3-PDO was observed as a primary byproduct $(37.7 \pm 13.2 \mathrm{C}$-mol\%) in TK52tz. Increased $3 \mathrm{HP}$ production might lead to the accumulation of intracellular $3 \mathrm{HPA}$, a precursor of $3 \mathrm{HP}$ as well as $1,3-\mathrm{PDO}$, and result in the overflow of flux toward 1,3-PDO production. We speculated that changing the aldehyde dehydrogenase to the superior enzyme [17], or disruption of $y q h D$, whose product converts 3HPA to 1,3-PDO, would increase 3HP production and decrease 1,3-PDO production.

The single knockout strains of tpiA and $z w f$, TK52t and TK52z, respectively, were constructed to analyze the 
effects of the knockout of each gene. Metabolic simulation predicted that the $z w f$ knockout would not affect metabolism, but the tpiA knockout would alter metabolic flux into the Entner-Doudoroff pathway and decrease the growth rate. Similar culture results between TK52z and TK52 (Figure 1A-1D, Table 1) suggest that the flux into the oxidative pentose phosphate pathway was small, and the deletion of $z w f$ had a small impact on metabolism in this condition, as predicted by the simulation. The culture results of TK52t and TK52tz were similar (Figure 1E-1H, Table 1). The $3 \mathrm{HP}$ yield of TK52t was increased by 3.2 -fold relative to TK52 (14.7 $\pm 7.0 \mathrm{C}$-mol\%) and slightly reduced when compared to TK52tz. TK52t produced 1,3-PDO $(22.9 \pm 5.2 \mathrm{C}-\mathrm{mol} \%)$ as a byproduct, as predicted by metabolic simulation. Acetate production by the tpiA knockout was also predicted, however measurements of acetate production $(8.2 \pm 9.9 \mathrm{C}-\mathrm{mol} \%)$ in TK52t contained significant variation, thus it was difficult to compare the experimental results with the results from the metabolic simulation.

The increased 3HP yield in the tpiA knockout, which was not predicted by metabolic simulation, might be due to the conversion of glycerol to glycerol 3-phosphate by glycerol kinase, which may have had a higher activity than the glycerol dehydrogenase that converts glycerol to DHA [40]. This would lead to increased flux into the methylglyoxal pathway, as indicated by elevated methylglyoxal levels (Figure 1F), resulting in similar culture results for TK52tz (Figure $1 \mathrm{H}$ ), such as 3HP production.

\section{Further increase in 3HP production by yqhD deletion, based on measurement of 1,3-PDO production}

$3 \mathrm{HP}$ production was successfully increased in TK52tz, however a high yield of 1,3-PDO was also produced (37.7 $\pm 13.2 \mathrm{C}$-mol\%). $y q h D$, which is involved in 1,3-PDO biosynthesis, was deleted in TK52tz, generating the strain TK52tzy, in order to decrease 1,3-PDO production and further increase $3 \mathrm{HP}$ production. As a result, the $3 \mathrm{HP}$ yield of TK52tzy was increased 1.7-fold (33.9 $\pm 1.2 \mathrm{C}$-mol\%) and the 1,3-PDO yield was drastically reduced $(5.9 \pm 0.5 \mathrm{C}$-mol\%) relative to TK52tz (Figure 1I-J, Table 1). Compared to the parental strain, a 7.4-fold increase in 3HP yield was achieved in TK52tzy. Despite the deletion of $y q h D$, 1,3-PDO was still produced in TK52tzy $(5.9 \pm 0.5 \mathrm{C}$-mol\%), likely due to the presence of other alcohol dehydrogenases that might convert 3HPA to 1,3-PDO. The deletion of $y q h D$ increased the maximum concentration of extracellular methylglyoxal in TK52tzy $(0.60 \mathrm{mM}$ at maximum) relative to TK52tz (0.22 $\mathrm{mM}$ at maximum), since YqhD also utilizes methylglyoxal as a substrate [41]. The other culture results of TK52tzy, such as consumed glycerol and biomass yield, were similar to those of TK52tz (Table 1).
The culture results of TK52t and TK52tz revealed large variations in the production of 3HP, 1,3-PDO, and acetate and the consumption of glycerol (Figure 1E-H). On the other hand, the results from TK52tzy displayed smaller variations in these measurements (Figure 1I-J). Furthermore, TK52t and TK52tz produced a large amount of 1,3-PDO, which accompanies NADPH oxidation. The deletion of $y q h D$ in TK52tz decreased the magnitude of the error, suggesting that the high production of 1,3-PDO in TK52t and TK52tz might cause redox imbalance, resulting in the large variations in the measurements.

The 3HP yield of TK52tzy $(33.9 \pm 1.2 \mathrm{C}$-mol\%) was comparable to previous studies producing $3 \mathrm{HP}$ from glycerol via flask cultivation. Mohan et al. achieved 3HP yield of $39.0 \pm 0.01 \mathrm{C}$-mol\% by the optimization of culture conditions such as the initial culture medium $\mathrm{pH}$ [18]. Rathnasingh et al. achieved a yield of $40 \mathrm{C}-\mathrm{mol} \%$ by the expression of $\alpha$-ketoglutaric semialdehyde dehydrogenase instead of aldehyde dehydrogenase, and periodic supplementation with vitamin $B_{12}$, a coenzyme for glycerol dehydratase [17]. Jung et al. constructed an engineered $E$. coli strain by knocking out ackA, pta, and $y q h D$ to reduce byproduct generation and knocking out $g l p R$ and overexpressing $g l p F$ to enhance glycerol metabolism [42]. They achieved high 3HP production $(42 \mathrm{~g} / \mathrm{L})$ by fed-batch cultivation using a jar fermenter, but the 3HP yield was lower (26.2 C-mol\%) than that achieved in this study. In previous studies [17,18], acetate was a major byproduct, as it was in this study, and higher yields of succinate, lactate, and ethanol were also produced. Succinate, lactate, and ethanol production might serve to oxidize the excess NADH that accompanies 3HP production and glycerol catabolism via glycolysis, since production of these metabolites requires NADH as a reducing agent. Conversely, the yields of these metabolites were small in TK52tzy. This might be because the deletion of tpiA and $z w f$ prevented flux into glycolysis, reducing excess NADH production, as predicted by metabolic simulation.

In this study, metabolic simulation was performed under the conditions of OUR/GUR $=0.67 \quad$ (OUR $=10 \mathrm{mmol} /$ (g DC•h) and GUR = $15 \mathrm{mmol} /(\mathrm{g}$ DC•h)), and additional simulations were also performed under other various OUR/GUR conditions (data not shown). Given the 3HP yield of TK52tzy $(33.9 \pm 1.2 \mathrm{C}$-mol\%), the metabolic state of the cell in this study was estimated under the condition of $\mathrm{OUR} / \mathrm{GUR} \approx 1$, by the metabolic simulation. Although the adjustment of oxygen supply to the simulation result was difficult using flask cultivation (oxygen transfer coefficient, $\mathrm{k}_{\mathrm{L}} \mathrm{a}$, of a shaking flask is $10-1001 / \mathrm{h}$ ), the metabolic simulation was used effectively for improving $3 \mathrm{HP}$ production. Further increases in 3HP production can be expected by the optimization of aeration conditions using a bioreactor. 
Metabolic simulation is a powerful tool for the design of metabolic engineering strategies to improve target production and has been used successfully in many studies. Metabolic simulation using FBA is simply based on the assumption of steady state metabolism without considering the complex cellular mechanisms such as enzyme activity and regulation. This can cause discrepancies between the simulation and experimental results, i.e., the unpredicted production of 1,3-PDO in the present study. Thus, examination of the differences between experimental and simulation results, and conformation of the constructed strains to the simulated metabolic state, is important for further improvement of target production.

\section{Conclusions}

In conclusion, the production of $3 \mathrm{HP}$ from glycerol in $E$. coli was improved by the modification of central metabolism, based on metabolic simulation and experimental validation of the engineered strains. Gene knockout simulations using the genome-scale metabolic model identified tpiA and $z w f$ as candidates to be modified and in this double knockout strain, TK52tz, 3HP yield was increased 4.4-fold relative to the TK52 parent strain, as predicted. Increased extracellular methylglyoxal in TK52tz suggested that glycerol catabolism through the methylglyoxal pathway was consistent with metabolic simulation. Since TK52tz produced 1,3-PDO as an abundant byproduct, $y q h D$, which encodes an enzyme involved in 1,3-PDO production, was deleted in TK52tz. The resulting strain, TK52tzy, exhibited a 1.7 -fold increase in $3 \mathrm{HP}$ yield relative to TK52tz and a 7.4-fold increase (33.9 $\pm 1.2 \mathrm{C}$-mol\%) relative to TK52. The double knockout of tpiA and $z w f$ contributed to the reduced production of byproducts such as succinate and lactate that are associated with $\mathrm{NADH}$ oxidation, likely due to reduced $\mathrm{NADH}$ production by the inhibition of glycolysis. The successful increase in 3HP production, based on metabolic simulation, demonstrated the effectiveness of metabolic modeling in designing a metabolic engineering strategy. Moreover, experimental validation of the engineered strains and comparison with the simulation results provided additional modifications to the engineering strategy to increase target production.

\section{Methods}

\section{Strains and plasmids}

The strains used in this study are summarized in Table 4. The MG1655(DE3) strain was constructed based on E. coli MG1655, using the $\lambda$ DE3 Lysogenization Kit (Merck KGaA, Darmstadt, Germany). The 3HP-producing strain, TK52, was constructed as published previously [17]. Fragments containing dhaB1, dhaB2, and dhaB3, encoding for components of glycerol dehydratase, and the $g d r A$ and $g d r B$ genes, encoding for glycerol dehydratase reactivase, were amplified from the genomic DNA of $K$. pneumoniae subsp. pneumoniae (NBRC 14940), which was purchased from the National Institute of Technology and Evaluation (Tokyo, Japan). Fragments were generated by PCR using the primer pair 5'-CCGGAATTCATGAAAAG ATCAAAACGATTTGCAGTACT-3' and 5'-GTTAAG CTTGATCTCCCACTGACCAAAGCTGG-3' for dhaB (dhaB1, dhaB2, dhaB3) and $g d r A$ and the primer pair 5'-GAAAAGCTTGAGGGGGACCGTCATGTCGCTTTC ACCGCCAG-3' and 5'-GCGCTTAAGTCAGTTTCTCT CACTTAACGGC-3' for gdrB. The aldH gene was amplified from the genomic DNA of E. coli MG1655 with the primer pair 5'-GGAGGATCCATGAATTTTCATCATCT GGC-3' and 5'-TCGAAGCTTTCAGGCCTCCAGGCT TAT-3'. PCR was performed using KOD FX Neo (Toyobo Co., Ltd., Osaka, Japan). Each amplified fragment was treated with A-attachment mix (Toyobo Co., Ltd.), and then cloned into pGEM-T easy (Promega Co., Madison, WI, U.S.A.), followed by sequence confirmation by the BigDye Terminator v3.1 Cycle Sequencing Kit (Applied Biosystems, Inc., Foster City, CA, U.S.A.),

Table 4 The $E$. coli strains used in this study

\begin{tabular}{|c|c|c|}
\hline Strain & Genotype & Source \\
\hline BW25113 & $\mathrm{F}^{-}, \lambda^{-}, \operatorname{lacl}^{\mathrm{a}} \mathrm{rrn}_{\mathrm{T} 14} \Delta \mathrm{lac}_{\mathrm{WJ16}}$ hsdR514 $\triangle a r a B A-D_{\mathrm{AH} 33} \Delta r h a B A D_{\mathrm{LD} 78}$ & Datsenko and Wanner [43] \\
\hline JW3890 & The same as BW25113 but $\Delta t p i A: . k a n$ & Baba et al. [44] \\
\hline BW25113 $\Delta$ zWf:.cat & The same as BW25113 but $\Delta z w f:: c a t$ & Nakahigashi et al. [45] \\
\hline BW25113 $\Delta$ yqhD::tet & The same as BW25113 but $\Delta y q h D: . t e t$ & This study \\
\hline MG1655(DE3) & $\mathrm{F}^{-}, \lambda^{-}, r p h-1, \lambda(\mathrm{DE} 3[\mathrm{lacl}$ lacUV5-T7 gene I indl sam7 nin5]) & This study \\
\hline TK52 & MG1655(DE3) transformed with pTrc99A/aldH and pCDFDuet-1/dhaB-gdrAB & This study \\
\hline TK52t & The same as TK52 but $\triangle$ tpi::kan & This study \\
\hline TK52z & The same as TK52 but $\triangle$ zwf::cat & This study \\
\hline TK52tz & The same as TK52 but $\triangle$ tpiA::kan $\triangle$ zwf::cat & This study \\
\hline TK52tzy & The same as TK52 but $\Delta$ tpiA::kan $\Delta z w f::$ cat $\Delta y q h D::$ tet & This study \\
\hline
\end{tabular}


and the 3130 Genetic Analyzer (Applied Biosystems). The EcoRI-HindIII and HindIII-AflII fragments carrying $d h a B-g d r A$ and $g d r B$, respectively, on pGEM-T easy were cloned into the same restriction sites in pCDFDuet-1 (Merck KGaA), generating pCDFDuet-1/dhaB-gdrAB. In addition, the BamHI-HindIII fragment carrying aldH on pGEM-T easy was cloned into the pTrc99A expression vector (Pharmacia, Stockholm, Sweden), generating pTrc99A/ald $H$.

The knockout strains were constructed using Wanner's method [43] and P1 $k c$-mediated phage transduction [46]. For the deletion of $y q h D$, the disruption cassette, including the tetracycline resistance gene and homologous regions upstream and downstream of $y q h D$, was amplified from pKD13tet [45] by PCR with the primer pair 5'-GCA GATCGTTCTCTGCCCTCATAT TGGCCCA GCAAA GG GA GCAAGTAATGATTCCGGGGATCCG TCGACC-3' and 5'-CGAAAACGAAAGTTTGAGGCGTAAAAAGCT TAGCGGGCGGCTTCGTATATTGTAGGCTGGAGCTG CTTCG-3'. The disruption cassette was introduced into the BW25113/pKD46 strain to construct BW25113 4 qhD:: tet. To delete $z w f$, tpiA, and $y q h D$ in E. coli MG1655(DE3), P1 transduction was performed using P1 phage obtained from JW3890 [44], BW25113 $\Delta z w f:$ :cat and BW25113 $4 y q h D::$ tet strains, respectively. Finally, the plasmids pTrc99A/ald $H$ and pCDFDuet-1/dhaB-gdrAB, were introduced into MG1655(DE3) and the knockout strains to construct the 3HP-producing strains (Table 4).

\section{Medium and culture methods}

Pre-cultures were grown aerobically at $37^{\circ} \mathrm{C}$ overnight in Lennox medium (10 g/L tryptone, $5 \mathrm{~g} / \mathrm{L}$ yeast extract, $5 \mathrm{~g} / \mathrm{L} \mathrm{NaCl}, 1 \mathrm{~g} / \mathrm{L}$ glucose) containing $0.05 \mathrm{~g} / \mathrm{L}$ ampicillin and $0.05 \mathrm{~g} / \mathrm{L}$ streptomycin. Pre-cultures were transferred to the main culture with an initial optical density at $600 \mathrm{~nm}\left(\mathrm{OD}_{600}\right)$ of 0.02 . For the main culture, M9 medium $\left(17.1 \mathrm{~g} / \mathrm{L} \mathrm{Na}_{2} \mathrm{HPO}_{4} \cdot 12 \mathrm{H}_{2} \mathrm{O}, 3.0 \mathrm{~g} / \mathrm{L} \quad \mathrm{KH}_{2} \mathrm{PO}_{4}\right.$, $2.0 \mathrm{~g} / \mathrm{L} \mathrm{NH} \mathrm{NH}_{4} \mathrm{Cl} 0.5 \mathrm{~g} / \mathrm{L} \mathrm{NaCl}, 0.123 \mathrm{~g} / \mathrm{L} \mathrm{MgSO}_{4} \cdot 7 \mathrm{H}_{2} \mathrm{O}$, $0.00278 \mathrm{~g} / \mathrm{L} \quad \mathrm{FeSO}_{4} \cdot 7 \mathrm{H}_{2} \mathrm{O}, 0.0147 \mathrm{~g} / \mathrm{L} \quad \mathrm{CaCl}_{2} \cdot 2 \mathrm{H}_{2} \mathrm{O}$, $0.01 \mathrm{~g} / \mathrm{L}$ thiamine $\mathrm{HCl}$ ) supplemented with $2 \mathrm{~g} / \mathrm{L}$ yeast extract, $0.02 \mathrm{mM}$ cyanocobalamin, $0.1 \mathrm{mM}$ IPTG, $0.05 \mathrm{~g} / \mathrm{L}$ ampicillin, and $0.05 \mathrm{~g} / \mathrm{L}$ streptomycin was used. Cells were cultured in $500 \mathrm{~mL}$ Sakaguchi flasks containing $50 \mathrm{~mL}$ of the $\mathrm{M} 9$ medium at $37^{\circ} \mathrm{C}$ in a shaking incubator at $120 \mathrm{rpm}$ (MM-10, Taitec, Saitama, Japan).

\section{Analytical methods}

Cell growth was monitored by the measurement of $\mathrm{OD}_{600}$ using UV-mini 1240 (Shimadzu, Kyoto, Japan). Concentrations of glycerol, 3HP, 1,3-PDO, succinate, lactate, acetate, formate, and ethanol present in the supernatant of the culture broth were determined by an HPLC system (HPLC Prominence, Shimadzu) equipped with an Aminex HPX-87H column (Bio-Rad, Hercules,
CA, U.S.A.), a UV/vis detector (SPD-20A), and a refractive index detector (RID-10A). The column temperature was set to $65^{\circ} \mathrm{C}$, and $2 \mathrm{mM} \mathrm{H}_{2} \mathrm{SO}_{4}$ was used as the mobile phase with a flow rate of $0.5 \mathrm{~mL} / \mathrm{min}$. The flow cell temperature of the refractive index detector was set to $35^{\circ} \mathrm{C}$. The supernatant of the culture broth was obtained by centrifugation at $21,500 \times g$ for $5 \mathrm{~min}$ at $4^{\circ} \mathrm{C}$, and then filtered through a Millex HV $0.45-\mu$ m filter (Merck KGaA).

Methylglyoxal in the supernatant was quantified colorimetrically with 2,4-dinitrophenylhydrazine (2,4-DNPH) [47]. The reaction mixture containing $67 \mu \mathrm{L}$ of sample and $22 \mu \mathrm{L}$ of 2,4-DNPH solution (0.1\% 2,4-DNPH in $2 \mathrm{M} \mathrm{HCl}$ ) was incubated for $15 \mathrm{~min}$ at $30^{\circ} \mathrm{C}$ in a 96-well microtiter plate, and then $111 \mu \mathrm{L}$ of $10 \% \mathrm{NaOH}$ was added. After a 15-min incubation at room temperature, the absorbance at $544 \mathrm{~nm}$ was measured with a microplate reader (1420 ARVO, PerkinElmer Inc., Waltham, MA, U.S.A.).

\section{In silico metabolic simulation}

The genome-scale metabolic model of $E$. coli K-12, named iAF1260 [21], was used. iAF1260 contains 1,260 ORFs, 2,077 metabolic and transport reactions, and 1,038 unique metabolites. To simulate $3 \mathrm{HP}$ production by $E$. coli, seven reactions required for $3 \mathrm{HP}$ biosynthesis from glycerol were added to the iAF1260 model (Table 2), because neither the iAF1260 model nor the wildtype E. coli contained the 3HP biosynthesis pathway. The 3HP transport reaction was also added to the metabolic model as a proton symporter because the transport of a similar metabolite, D-lactate (2-hydroxypropionic acid), occurs through a proton symporter in the iAF1260 model. In the same manner, the 1,3-PDO transport reaction was added as a diffusion reaction, which is considered to utilize the 1,2-propandiol transporter in the iAF1260 model.

FBA was used to simulate the metabolic flux distribution in the genome-scale metabolic model [24,25]. Briefly, a pseudo-steady state of the metabolite profile was assumed, and the maximum and minimum ranges of the flux for each reaction were defined. These constraints provided a feasible space for the flux distribution in the metabolic model. To obtain an optimal flux distribution in the feasible space, an objective function was introduced and a linear programming technique was applied. This problem is represented by the following equation:

$$
\begin{aligned}
& \text { maximize }: c^{T} \cdot v \\
& \text { subject to }: \cdot S \cdot v=0 \\
& v_{\text {min }} \leq \nu \leq v_{\max }
\end{aligned}
$$

where $S$ represents the stoichiometric matrix of metabolites in metabolic reactions, and $v$ indicates a vector of flux for each metabolic reaction. The values $v_{\min }$ and $v_{\max }$ represent 
the minimum and maximum constraints of flux for each reaction, and $c$ is a vector that represents the objective function to be maximized or minimized. In this study, biomass production was used as the objective function to be maximized, with the assumption that cellular metabolism is self-organized to maximize growth rate. After the maximal biomass production flux was obtained, the $3 \mathrm{HP}$ production flux was maximized under the fixed biomass production flux, on the maximal value, to avoid undetermined 3HP production flux [29].

For metabolic simulation, glycerol was used as the sole carbon source, and the uptake rate was set to $15 \mathrm{mmol} /(\mathrm{g} \mathrm{DC} \bullet \mathrm{h})$. The oxygen uptake rate was set to $10 \mathrm{mmol} /(\mathrm{g} \mathrm{DC} \bullet \mathrm{h})$. Other external metabolites such as $\mathrm{CO}_{2}$ and $\mathrm{NH}_{3}$ were allowed to transport freely through the cell membrane. For gene knockout simulations, minimum and maximum fluxes of the corresponding reactions were set to zero. All simulations were performed using Matlab (MathWorks Inc., Natick, MA, U.S.A.) with a solver for linear programming, GLPK (GNU Linear Programming Kit, http://glpkmex.sourceforge.net/).

\begin{abstract}
Abbreviations
3HP: 3-hydroxypropionic acid; FBA: Flux balance analysis; 1,3propanediol: 1,3-PDO; 3HPA: 3-hydroxypropionaldehyde; GUR: Glycerol uptake rate; DC: Dry cell; OUR: Oxygen uptake rate; C-mol\%: Carbon-molar yield; DHAP: Dihydroxyacetone phosphate; GAP: Glyceraldehyde-3-phosphate; DHA: Dihydroxyacetone; 2,4-DNPH: 2,4-dinitrophenylhydrazine; G3P: Glycerol 3-phosphate; G6P: Glucose 6-phosphate; 6PG: 6-phospho-gluconate; 6PGL: 6-phospho-glucono-1,5-lactone; F6P: Fructose 6-phosphate; FBP: Fructose 1,6-bisphosphate; Ru5P: Ribulose 5-phosphate; R5P: Ribose 5-phosphate; S7P: Sedoheptulose 7-phosphate; X5P: Xylulose 5-phosphate; E4P: Erythrose 4-phosphate; MGO: Methylglyoxal; PEP: Phosphoenolpyruvate; Pyr: Pyruvate; AcCoA: Acetyl-CoA; AcetP: Acetyl phosphate; EtOH: Ethanol; Oxa: Oxaloacetate; Cit: Citrate; IsoCit: Isocitrate; a-KG: a-ketoglutarate; Suc: Succinate;

Fum: Fumarate; Mal: Malate; Glyox: Glyoxylate; Q: Ubiquinone; $\mathrm{QH}_{2}$ : Ubiquinol.
\end{abstract}

\section{Competing interests}

The authors have no conflicts of interest to declare.

\section{Authors' contributions}

$\mathrm{KT}$ carried out the strain construction and the culture experiments and drafted the manuscript. SO performed the metabolic simulation. KY participated in the design of the study, and drafted the manuscript. TH participated in the design of the study. ST carried out the strain construction. CF participated in the design of the study. HS conceived and supervised the study. All authors read and approved the final manuscript.

\section{Acknowledgements}

The authors thank Prof. Kenji Nakahigashi (Keio University) for kindly

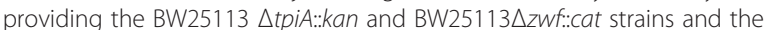
pKD13tet vector. The authors thank Prof. Fumio Matsuda (Osaka University) for his kind suggestions.

This work was supported by a Grant-in-Aid for Scientific Research (A) to HS (No. 24246134) and a Grant-in-Aid for Young Scientists (A) to CF (No. 23680030) from the Japan Society for the Promotion of Science. KT acknowledges financial aid (scholarship grant) supported by "Program for Leading Graduate Schools" of the Ministry of Education, Culture, Sports, Science and Technology, Japan.

\section{Author details}

'Department of Bioinformatic Engineering, Graduate School of Information Science and Technology, Osaka University, 1-5 Yamadaoka, Suita, Osaka 565-0871, Japan. ${ }^{2}$ Department of Bioengineering, Tokyo Institute of Technology, 4259 Nagatsuta-cho, Midori-ku, Yokohama 226-8501, Japan.
${ }^{3}$ Quantitative Biology Center, RIKEN, 6-2-3 Furuedai, Suita, Osaka 565-0874, Japan.

Received: 11 March 2014 Accepted: 21 April 2014

Published: 7 May 2014

\section{References}

1. Valdehuesa KN, Liu H, Nisola GM, Chung WJ, Lee SH, Park SJ: Recent advances in the metabolic engineering of microorganisms for the production of 3-hydroxypropionic acid as C3 platform chemical. Appl Microbiol Biotechnol 2013, 97:3309-3321.

2. Kumar $\mathrm{V}$, Ashok S, Park S: Recent advances in biological production of 3-hydroxypropionic acid. Biotechnol Adv 2013, 31:945-961.

3. Wang Q, Yang P, Liu C, Xue Y, Xian M, Zhao G: Biosynthesis of poly(3hydroxypropionate) from glycerol by recombinant Escherichia coli. Bioresour Technol 2013, 131:548-551.

4. Andreessen B, Steinbuchel A: Biosynthesis and biodegradation of 3-hydroxypropionate-containing polyesters. Appl Environ Microbiol 2010, 76:4919-4925.

5. Werpy T, Petersen G: Top Value Added Chemicals from Biomass: Volume I - Results of Screening for Potential Candidates from Sugars and Synthesis Gas. ; 2004

6. Bozell JJ, Petersen GR: Technology development for the production of biobased products from biorefinery carbohydrates - the US Department of Energy's "Top 10" revisited. Green Chem 2010, 12:539.

7. da Silva GP, Mack M, Contiero J: Glycerol: a promising and abundant carbon source for industrial microbiology. Biotechnol Adv 2009, 27:30-39.

8. Ito T, Nakashimada Y, Senba K, Matsui T, Nishio N: Hydrogen and ethanol production from glycerol-containing wastes discharged after biodiesel manufacturing process. J Biosci Bioeng 2005, 100:260-265.

9. Mattam AJ, Clomburg JM, Gonzalez R, Yazdani SS: Fermentation of glycerol and production of valuable chemical and biofuel molecules. Biotechnol Lett 2013, 35:831-842.

10. Ashok S, Mohan Raj S, Ko Y, Sankaranarayanan M, Zhou S, Kumar V, Park S: Effect of puuC overexpression and nitrate addition on glycerol metabolism and anaerobic 3-hydroxypropionic acid production in recombinant Klebsiella pneumoniae $\Delta$ glpKAdhaT. Metab Eng 2013, 15:10-24.

11. Ashok S, Raj SM, Rathnasingh C, Park S: Development of recombinant Klebsiella pneumoniae $\Delta$ dhaT strain for the co-production of 3-hydroxypropionic acid and 1,3-propanediol from glycerol. Appl Microbiol Biotechnol 2011, 90:1253-1265.

12. Ashok S, Sankaranarayanan M, Ko Y, Jae KE, Ainala SK, Kumar V, Park S: Production of 3-hydroxypropionic acid from glycerol by recombinant Klebsiella pneumoniae $\Delta d h a T \Delta y q h D$ which can produce vitamin $B_{12}$ naturally. Biotechnol Bioeng 2013, 110:511-524.

13. Luo LH, Seo JW, Heo SY, Oh BR, Kim DH, Kim CH: Identification and characterization of Klebsiella pneumoniae aldehyde dehydrogenases increasing production of 3-hydroxypropionic acid from glycerol. Bioprocess Biosyst Eng 2013, 36:1319-1326.

14. Zhou S, Ashok S, Ko Y, Kim DM, Park S: Development of a deletion mutant of Pseudomonas denitrificans that does not degrade 3-hydroxypropionic acid. Appl Microbiol Biotechnol 2014, doi:10.1007/s00253-014-5562-5.

15. Zhou S, Catherine C, Rathnasingh C, Somasundar A, Park S: Production of 3-hydroxypropionic acid from glycerol by recombinant Pseudomonas denitrificans. Biotechnol Bioeng 2013, 110:3177-3187.

16. Raj SM, Rathnasingh C, Jo J-E, Park S: Production of 3-hydroxypropionic acid from glycerol by a novel recombinant Escherichia coli BL21 strain. Process Biochem 2008, 43:1440-1446.

17. Rathnasingh C, Raj SM, Jo JE, Park S: Development and evaluation of efficient recombinant Escherichia coli strains for the production of 3-hydroxypropionic acid from glycerol. Biotechnol Bioeng 2009, 104:729-739.

18. Mohan Raj S, Rathnasingh C, Jung WC, Park S: Effect of process parameters on 3-hydroxypropionic acid production from glycerol using a recombinant Escherichia coli. Appl Microbiol Biotechnol 2009, 84:649-657.

19. Kim K, Kim SK, Park YC, Seo JH: Enhanced production of 3-hydroxypropionic acid from glycerol by modulation of glycerol metabolism in recombinant Escherichia coli. Bioresour Technol 2014, 156C:170-175.

20. Ida Y, Hirasawa T, Furusawa C, Shimizu H: Utilization of Saccharomyces cerevisiae recombinant strain incapable of both ethanol and glycerol biosynthesis for anaerobic bioproduction. Appl Microbiol Biotechnol 2013, 97:4811-4819. 
21. Feist AM, Henry CS, Reed JL, Krummenacker M, Joyce AR, Karp PD, Broadbelt LU, Hatzimanikatis V, Palsson BO: A genome-scale metabolic reconstruction for Escherichia coli K-12 MG1655 that accounts for 1260 ORFs and thermodynamic information. Mol Syst Biol 2007, 3:121.

22. Yoshikawa K, Kojima Y, Nakajima T, Furusawa C, Hirasawa T, Shimizu H: Reconstruction and verification of a genome-scale metabolic model for Synechocystis sp. PCC6803. Appl Microbiol Biotechnol 2011, 92:347-358.

23. Shinfuku Y, Sorpitiporn N, Sono M, Furusawa C, Hirasawa T, Shimizu H: Development and experimental verification of a genome-scale metabolic model for Corynebacterium glutamicum. Microb Cell Fact 2009, 8:43.

24. Orth JD, Thiele I, Palsson BO: What is flux balance analysis? Nat Biotechnol 2010, 28:245-248.

25. Oberhardt MA, Palsson BO, Papin JA: Applications of genome-scale metabolic reconstructions. Mol Syst Biol 2009, 5:320.

26. Feist AM, Palsson BO: The growing scope of applications of genome-scale metabolic reconstructions using Escherichia coli. Nat Biotechnol 2008, 26:659-667.

27. Price ND, Reed JL, Palsson BO: Genome-scale models of microbial cells: evaluating the consequences of constraints. Nat Rev Microbiol 2004 2:886-897.

28. Burgard AP, Pharkya P, Maranas CD: Optknock: a bilevel programming framework for identifying gene knockout strategies for microbial strain optimization. Biotechnol Bioeng 2003, 84:647-657.

29. Ohno S, Furusawa C, Shimizu H: In silico screening of triple reaction knockout Escherichia coli strains for overproduction of useful metabolites. J Biosci Bioeng 2013, 115:221-228.

30. Ohno S, Shimizu H, Furusawa C: FastPros: screening of reaction knockout strategies for metabolic engineering. Bioinformatics 2013, 30:981.

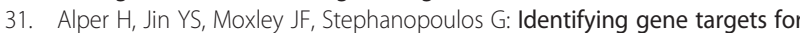
the metabolic engineering of lycopene biosynthesis in Escherichia coli. Metab Eng 2005, 7:155-164.

32. Park JH, Lee KH, Kim TY, Lee SY: Metabolic engineering of Escherichia coli for the production of L-valine based on transcriptome analysis and in silico gene knockout simulation. Proc Natl Acad Sci U S A 2007, 104:7797-7802.

33. Lee SJ, Lee DY, Kim TY, Kim BH, Lee J, Lee SY: Metabolic engineering of Escherichia coli for enhanced production of succinic acid, based on genome comparison and in silico gene knockout simulation. App/ Environ Microbiol 2005, 71:7880-7887.

34. Zhou L, Zuo ZR, Chen XZ, Niu DD, Tian KM, Prior BA, Shen W, Shi GY, Singh S, Wang ZX: Evaluation of genetic manipulation strategies on D-lactate production by Escherichia coli. Curr Microbiol 2011, 62:981-989.

35. Hasunuma T, Sanda T, Yamada R, Yoshimura K, Ishii J, Kondo A: Metabolic pathway engineering based on metabolomics confers acetic and formic acid tolerance to a recombinant xylose-fermenting strain of Saccharomyces cerevisiae. Microb Cell Fact 2011, 10:2

36. Li H, Liao JC: Engineering a cyanobacterium as the catalyst for the photosynthetic conversion of $\mathrm{CO}_{2}$ to 1,2-propanediol. Microb Cell Fact 2013, 12:4.

37. Cooper RA: Metabolism of methylglyoxal in microorganisms. Annu Rev Microbiol 1984, 38:49-68.

38. Schuetz R, Kuepfer L, Sauer U: Systematic evaluation of objective functions for predicting intracellular fluxes in Escherichia coli. Mol Syst Biol 2007, 3:119.

39. Totemeyer S, Booth NA, Nichols WW, Dunbar B, Booth IR: From famine to feast: the role of methylglyoxal production in Escherichia coli. Mol Microbiol 1998, 27:553-562

40. Durnin G, Clomburg J, Yeates Z, Alvarez PJ, Zygourakis K, Campbell P, Gonzalez R: Understanding and harnessing the microaerobic metabolism of glycerol in Escherichia coli. Biotechnol Bioeng 2009, 103:148-161.

41. Jarboe LR: YqhD: a broad-substrate range aldehyde reductase with various applications in production of biorenewable fuels and chemicals. Appl Microbiol Biotechnol 2011, 89:249-257.

42. Jung WS, Kang JH, Chu HS, Choi IS, Cho KM: Elevated production of 3-hydroxypropionic acid by metabolic engineering of the glycerol metabolism in Escherichia coli. Metab Eng 2014, 23C:116-122.

43. Datsenko KA, Wanner BL: One-step inactivation of chromosomal genes in Escherichia coli K-12 using PCR products. Proc Natl Acad Sci U S A 2000, 97:6640-6645.

44. Baba T, Ara T, Hasegawa M, Takai Y, Okumura Y, Baba M, Datsenko KA, Tomita M, Wanner BL, Mori H: Construction of Escherichia coli K-12 in-frame, single-gene knockout mutants: the Keio collection. Mol Syst Biol 2006, 2:2006-0008.

45. Nakahigashi K, Toya Y, Ishii N, Soga T, Hasegawa M, Watanabe H, Takai Y, Honma M, Mori H, Tomita M: Systematic phenome analysis of Escherichia coli multiple-knockout mutants reveals hidden reactions in central carbon metabolism. Mol Syst Biol 2009, 5:306.

46. Thomason LC, Costantino N, Court DL: E. coli genome manipulation by P1 transduction. Curr Protoc Mol Biol 2007, 1:1-17.

47. Cooper RA, Anderson A: The formation and catabolism of methylglyoxal during glycolysis in Escherichia coli. FEBS Lett 1970, 11:273-276.

doi:10.1186/1475-2859-13-64

Cite this article as: Tokuyama et al:: Increased 3-hydroxypropionic acid production from glycerol, by modification of central metabolism in Escherichia coli. Microbial Cell Factories 2014 13:64.

\section{Submit your next manuscript to BioMed Central and take full advantage of:}

- Convenient online submission

- Thorough peer review

- No space constraints or color figure charges

- Immediate publication on acceptance

- Inclusion in PubMed, CAS, Scopus and Google Scholar

- Research which is freely available for redistribution

C BioMed Central 